\title{
MAGNETIC PROPERTIES OF $\mathrm{Sn}_{1-x} \mathrm{Gd}_{x} \mathrm{Te}$ $(0.002 \leq x \leq 0.09)^{*}$
}

T. Story, M. Arciszewska, W. Dobrowolski, Z. Golacki, M. Górska, E. Grodzicka, A. Eusakowski, E. Dynowska and B. WitkowsKa

Institute of Physics, Polish Academy of Sciences

Al. Lotników 32/46, 02-668 Warsaw, Poland

\begin{abstract}
Magnetic, transport and structural properties of bulk crystals of $\mathrm{Sn}_{1-x} \mathrm{Gd}_{x} \mathrm{Te}$ with $\mathrm{Gd}$ content $0.002<x<0.09$ and varying carrier concentrations obtained by an isothermal annealing were studied in the temperature range $T=1.5-80 \mathrm{~K}$. We found the effect of resonant increase in antiferromagnetic spin-spin exchange interactions in the crystals with $0.025 \leq x \leq 0.05$. No effect was found in crystals either with higher $(x>0.05)$ or with lower $(x<0.025) \mathrm{Gd}$ concentration. The observed Gd composition dependence of the magnetic and transport properties of $\mathrm{SnGdTe}$ can be explained in a proposed model relating these experimental properties to the $\mathrm{Gd}$ composition induced shift of the position of $\mathrm{Gd}^{3+/ 2+}$ level with respect to the top of the valence band of SnGdTe.
\end{abstract}

PACS numbers: $75.20 . \mathrm{Ck}, 75.30$.Et

It was recently discovered that both magnetic and transport properties of $\mathrm{Sn}_{1-x} \mathrm{Gd}_{x} \mathrm{Te}$ semimagnetic semiconductor depend strongly on the position of the Fermi level with respect to the top of the valence band. In the crystals with Gd composition $x=0.045$ the antiferromagnetic $f-f$ spin interactions were found to be strongly enhanced in the samples with carrier concentration $p=$ $(3 \pm 0.5) \times 10^{20} \mathrm{~cm}^{-3}$. In the very same samples the strong increase in carrier mobility was also observed [1-3]. No such effects were found in the crystals with $x=0.057$. These experimental findings were explained in a model assuming that the Gd $5 d^{1}$ energy states are located at the energy $E_{0}$ close to the Fermi level of $\mathrm{SnGdTe}$. This creates the $\mathrm{Gd}^{2+}-\mathrm{Gd}^{3+}$ mixed valence system. The $5 d$ orbitals of $\mathrm{Gd}$ are much less localized than the $4 f$ orbitals and provide the effective channel for two-stage spin-spin exchange interaction mechanism, proceeding via an intraion $4 f-5 d$ and an interion $5 d-5 d$ exchange interaction. This mechanism is expected to be resonantly enhanced for $E_{\mathrm{F}} \approx E_{0}$ [4]. Its strength depends on the density of states at the Fermi level. In the model, the dramatic composition dependence of the magnetic and transport properties of SnGdTe is related to the composition

*This work was supported in part by the Committee for Scientific Research (Poland) by grant 2 P03 B 10308. 
dependent shift of the top of the valence band of SnGdTe with respect to the $E_{0}$ level of Gd. The aim of our paper is to study these effects in wide Gd concentration range in order to verify the above-mentioned model.

We studied the bulk crystals of $\mathrm{Sn}_{1-x} \mathrm{Gd}_{x} \mathrm{Te}$ with $\mathrm{Gd}$ content $0.002<$ $x<0.09$ and varying carrier concentrations obtained by an isothermal annealing. The ac magnetic susceptibility was measured over the temperature range $T=1.5-80 \mathrm{~K}$ by mutual inductance bridge applying the ac magnetic field of $40 \mathrm{Gs}$. The Hall effect and the electric conductivity were studied in the temperature range $T=4-300 \mathrm{~K}$ by standard 4-probe dc method. Our samples were examined by microprobe analysis and by the Debye powder diffraction method. The crystals containing up to 9 at.\% of Gd were found to be single-phase and homogeneous.

By applying the standard analysis of the temperature dependence of the magnetic susceptibility of our crystals we determined the paramagnetic Curie temperature $\Theta$ for $\mathrm{SnGdTe}$ samples with different Gd composition and various carrier concentrations. The paramagnetic Curic temperature is proportional to the sum of all interspin interactions and provides the simple experimental measure of the strength of the interspin interactions in the system.

We have observed the effect of the strong carrier concentration dependence of the paramagnetic Curie temperature in a few samples with $0.025<x<0.05$. An example of such a behavior is presented in Fig. 1a. The sample with the lowest Gd concentration which did show this behavior had $x=0.025$. The very different experimental result was observed for the samples with $x=0.022$ (shown in Fig. 1b) where the annealing (changing carrier concentration) had only minor influence on the magnetic properties of the sample. In agreement with the previous results the increase in the paramagnetic Curie temperature was also not observed for the samples with $0.05<x<0.07$.

A model of the band structure of $\mathrm{Sn}_{1-x} \mathrm{Gd}_{x}$ Te providing the basis for the consistent explanation of both previous and new experimental results on magnetic properties of SnGdTe is presented in Fig. 2. The key element of this model is the dependence of the location of $\mathrm{Gd} 5 d$ electron states (the $E_{0}, \mathrm{Gd}^{2+/ 3+}$ level) with respect to the top of the valence band. The assumed linear dependence of the energy separation between the $E_{0}$ level and the top of the valence band on the composition of $\mathrm{Gd}$ is quite plausible. It is known that the mixed crystals of IV-VI semiconductors and rare-earth chalcogenides reveal the strong increase in the energy gap with the increasing content of the rare-earth element (the rate of about $40 \mathrm{meV} /$ at.\% [5]). The deep energy states related to $\mathrm{Gd}$ are expected to provide a critain reference energy level [6]. On has to notice, however, that the definite curfirmation of this dependence can n!ly be obtained from the analysis of oprical proprosties of $\mathrm{Sn}_{1-x} \mathrm{Gd}_{x} \mathrm{Te}$. These experimental data are not available yet. Also the analysis of the optical data will be strongly obscured by the semi-metallic charactcr of SnGdTe crystals leading to the spectral overlap between the fundamental alimention and the absorption on free carriers.

Within the frame of the model presented above the existence of the low concentration limit for the observation of the resonant increase in the interspin interactions is a simple consequence of the shift of the $E_{0}$ level deep into the valence 

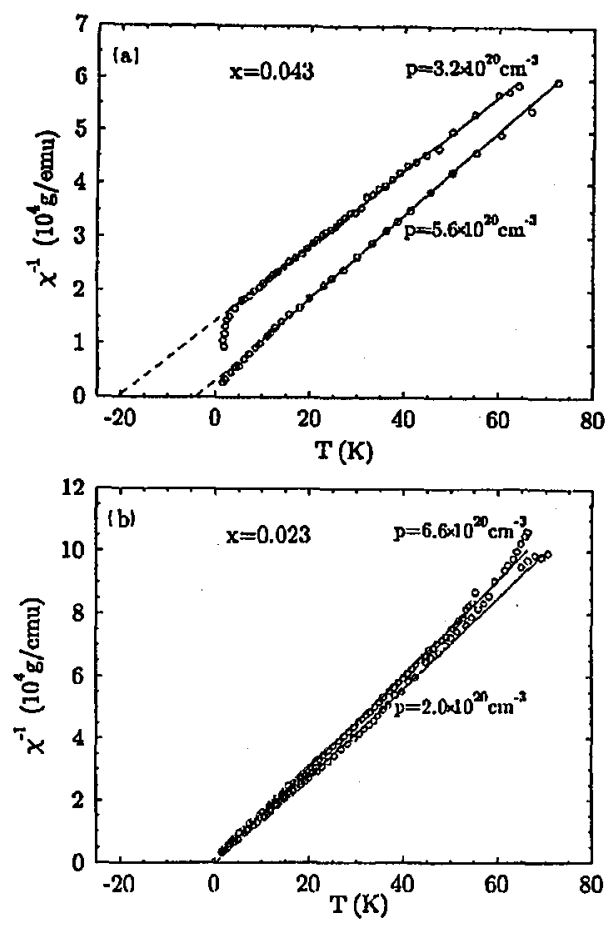

Fig. 1. The temperature dependence of the inverse magnetic susceptibility of the crystals of $\mathrm{Sn}_{1-x} \mathrm{Gd}_{x} \mathrm{Te}$ with different $\mathrm{Gd}$ concentrations and varying concentration of carriers.

band, into the energy range not covered by the Fermi level positions possible for SnGdTe. Therefore, it is not possible to reach the resonance $E_{0} \approx E_{\mathrm{F}}$ and, consequently, no carrier concentration dependence of the spin-spin interaction is expected. With the very same composition dependence of the $E_{0}$ level one expects that this level will shift up and will cross the top of the $\Sigma$-band at $x \approx 0.05$. For $x>0.05$ the $E_{0}$ level is resonant with the $L$-band of light holes only. The resonance condition $E_{\mathrm{F}} \approx E_{0}$ can be fulfilled but the density of states at the Fermi level is an order of magnitude smaller than in the case of the $\Sigma$-band of heavy holes. This strongly reduces the strength of the interaction.

The most interesting situation is expected for the mixed-valence regime observed for $0.025<x<0.05$ where both conditions for the effective $4 f-5 d-4 f$ exchange interaction are fulfilled. This is the composition range studied in our previous papers $[1,2]$ where the effect of the resonant enhancement of the $f-f$ exchange interactions was first observed.

According to the model proposed above an interesting new situation is also expected in the case of $x>0.1$. The location of the $E_{0}$ level in the energy gap of SnGdTe indicates a possible semi-insulating state of $\mathrm{SnGdTe}$ at low temperatures, with no free carriers participating in the interspin exchange interactions. In the 


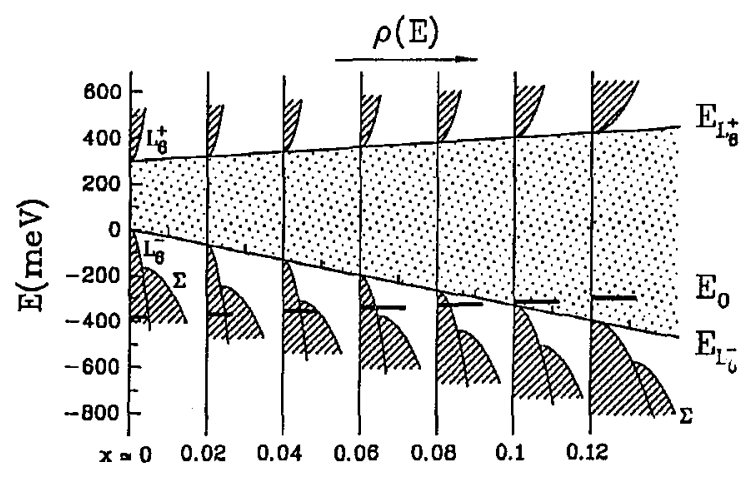

Fig. 2. A model of $\mathrm{Gd}$ composition dependence of the position of $\mathrm{Gd} 5 d$ electron states (the $E_{0}$ level) with respect to the top of the valence band of SnGdTe. The top of the $\Sigma$-band of heavy holes is located approximately $0.2 \mathrm{eV}$ below the top of the $L$-band of light holes.

opposite limit, i.e. $x \approx 0$, the $E_{0}$ level of $\mathrm{Gd}$ is expected to be located well below the Fermi level of SnGdTe. As a consequence, all the Gd ions will be in the 2+ $\left(4 f^{7} 5 d^{1}\right)$ charge state. This creates quite unusual situation with the spin of an ion being built from the spins of two inner unfilled atomic shells ( $4 f$ and $5 d)$. This conclusion can be verified by electron paramagnetic resonance measurements.

In conclusion, we found the effect of resonant increase in antiferromagnetic spin-spin exchange interactions in the $\mathrm{Sn}_{1-x} \mathrm{Gd}_{x} \mathrm{Te}$ crystals with $0.025 \leq x \leq$ 0.05 . No effect was found in crystals either with higher $(x>0.05)$ or with lower $(x<0.025) \mathrm{Gd}$ concentration. The observed Gd composition dependence of the magnetic properties of SnGdTe can be explained in a model assuming that with the increasing $\mathrm{Gd}$ composition the mutual position of the top of the valence band and the $E_{0}$ level changes in such a way that the $E_{0}$ level moves closer to the top of the band.

\section{References}

[1] T. Story, M. Górska, M. Arciszewska, E. Grodzicka, Z. Gołacki, R.R. Gałązka, J. Magn. Magn. Mater. 140-144, 2041 (1995).

[2] M. Górska, T. Story, M. Arciszewska, E. Grodzicka, Z. Gołacki, A. Eusakowski, Acta Phys. Pol. A 87, 197 (1995).

[3] W. Dobrowolski, E. Grodzicka, T. Story, Z. Gołacki, R.R. Galazzka, in: Narrow Gap Semiconductors 1995, Int. Phys. Conf. Ser., Vol. 144, Ed. J.L. Reno, IOP Publishing, Bristol 1995, p. 125.

[4] A. Eusakowski, Acta Phys. Pol. A 88, 1018 (1995).

[5] G. Bauer, H. Pascher, W. Zawadzki, Semicond. Sci. Technol. 7, 703 (1992).

[6] J.M. Langer, H. Heinrich, Phys. Rev. Lett. 55, 1414 (1985). 